\title{
Mensuração de área de úlceras por pressão por meio dos softwares Motic e do AutoCAD ${ }^{\circledast}$
}

\author{
Evaluation of pressure ulcers area using the softwares Motic and AutoCAD ${ }^{\circledR}$
}

Mensuración de área de úlceras por presión por medio de los programas informáticos Motic y AutoCAD ${ }^{\circledast}$.

\author{
Camila Letícia Dias dos Reis', Janaína Mortosa Cavalcante', Edvar Ferreira da Rocha Júnior", \\ Rinaldo Souza Neves"II, Levy Aniceto Santana ${ }^{\text {IV }}$, Renato da Veiga Guadagnin ${ }^{\mathrm{V}}$, Lourdes Mattos Brasil ${ }^{\mathrm{VI}}$ \\ ' Secretaria de Estado de Saúde do Distrito Federal. Brasília-DF, Brasil. \\ "Hospital do Coração do Brasil, Serviço de Fisioterapia. Brasília-DF, Brasil. \\ "I' Secretaria de Estado de Saúde do Distrito Federal, Fundação de Ensino e Pesquisa em Ciências da Saúde. Brasília-DF, Brasil. \\ Iv Secretaria de Estado de Saúde do Distrito Federal, Fundação de Ensino e Pesquisa em Ciências da Saúde. Universidade \\ Católica de Brasília. Brasília-DF, Brasil. \\ ${ }^{\vee}$ Universidade Católica de Brasília. Brasília-DF, Brasil. \\ ${ }^{v}$ Universidade de Brasília, Faculdade UnB-Gama, Curso de Engenharia Eletrônica. Brasília-DF, Brasil.
}

Submissão: 18-04-2010 Aprovação: 21-05-2012

\section{RESUMO}

A úlcera por pressão é uma lesão parcial ou total das camadas da pele em determinadas regiões do corpo e seu processo de cicatrização pode ser acompanhado por meio de processamento de imagens. A análise da área da úlcera por pressão é relevante para avaliação da sua evolução e de sua resposta a procedimentos terapêuticos. Os softwares Motic e AutoCAD ${ }^{\circ}$ permitem avaliar a área de regiões de uma imagem através da marcação do seu contorno. Valendo-se dessa técnica, 35 voluntários determinaram as áreas de duas úlceras por pressão grau III pelos dois tipos de software. Concluiu-se que eles apresentam resultados clinicamente concordantes e podem assim ser utilizados para acompanhamento da evolução da cicatrização de úlceras por pressão.

Descritores: Úlcera por pressão; Fotografia; Software; Processamento de imagem assistida por computador; Cicatrização de feridas.

\begin{abstract}
Pressure ulcer is a lesion that affects skin layers in some regions of the body and its healing can be followed up using image processing. The analysis of pressure ulcer area is relevant to evaluate its evolution and response to therapeutic procedures. Such areas can be evaluated through contour marking with the softwares Motic and AutoCAD ${ }^{\circ}$. In this study 35 volunteers computed areas from two grade III pressure ulcers using these instruments. It was possible to conclude that results are clinically equivalent and so can be considered to follow up healing evolution from pressure ulcers.

Key words: Pressure ulcer; Photography; Software; Image processing, computer-assisted; Wound healing.
\end{abstract}

\section{RESUMEN}

Úlcera por presión es una lesión parcial o total de las capas de la piel en determinadas regiones del cuerpo y su cicatrización puede ser acompañada por el procesamiento de imágenes. El análisis de área de la úlcera por presión es pertinente para evaluar su evolución y respuesta a procedimientos terapéuticos. Los programas informáticos Motic y AutoCAD ${ }^{\oplus}$ permiten evaluar la área de una imagen por medio de la delimitación de su circunferencia. Con esta técnica, 35 voluntarios determinaron las áreas de dos ulceras por presión de grado III en los dos programas informáticos. Se concluye que ellos presentan resultados clínicamente concordantes y pueden ser utilizados para el acompañamiento de la evolución de la cicatrización de las úlceras por presión. Palabras clave: Úlcera por presión; Fotografía; Programas informáticos; Procesamiento de imagen asistida por computador; Cicatrización de heridas. 


\section{INTRODUÇÃO}

A Úlcera por Pressão (UP) constitui uma lesão parcial ou total das camadas da pele e, freqüentemente, não se limita às camadas superficiais, atingindo o tecido subcutâneo, a fáscia e o tecido muscular, em determinadas regiões do corpo com menor quantidade de massa muscular e presença de proeminências ósseas em contato com uma superfície externa ${ }^{(1,2)}$.

Diversas escalas podem predizer o risco de formação de UP entre as quais, a mais citada na literatura, é a Escala de Braden, que avalia seis fatores de risco ou subescalas, sendo três referentes a determinantes clínicos de exposição para pressão intensa e prolongada - percepção sensorial, atividade e mobilidade; e três mensurando a tolerância do tecido à pressão - umidade, nutrição, fricção e cisalhamento. Ao final da avaliação, chega-se a uma pontuação, classificando o paciente como de risco elevado, risco moderado e risco mínimo para desenvolvimento de UP(3).

O risco de desenvolvimento de UP é maior em pacientes hospitalizados, tratados em domicílio ou em centros de reabilitação, portadores de patologias crônicas, tais como Acidente Vascular Encefálico (AVE), câncer, lesão medular quando estão em Unidade de Terapia Intensiva (UTI) ou idosos portadores de patologias crônicas $^{(4)}$.

As UPs podem ser classificadas de acordo com a profundidade, em relação à extensão da parede tissular envolvida: grau I - comprometimento da epiderme com eritema em pele íntegra; grau II - perda parcial da pele, envolvendo a epiderme e/ ou derme; grau III - comprometimento da epiderme, derme e tecido subcutâneo; e grau IV - comprometimento da epiderme, derme, tecido subcutâneo e tecidos mais profundos como músculos, tendões e ossos ${ }^{(5)}$.

A evolução do processo de cicatrização pode ser acompanhada por meio da mensuração da UP realizada por métodos não invasivos: medida simples (comprimento x largura em centímetros com uso de régua após desbridamento); decalque (traçar o formato da úlcera em material transparente e posteriormente colocando-o sobre um papel quadriculado, contando-se o número de quadrados inteiros para obtenção da área); fotografia (evidência visual do aspecto da úlcera, exigindo a repetição do procedimento em intervalos regulares sob o mesmo ângulo, luminosidade e distância focal constante); e sistemas computadorizados por meio de software para análise digital de imagens ${ }^{(5)}$.

Devido às desvantagens e limitações apresentadas pelos métodos invasivos, têm sido desenvolvidos e aperfeiçoados métodos não invasivos de mensuração da profundidade, volume, área e cor da UP, que utilizam fotografias e software para o processamento de imagens e mensuração de UPs ${ }^{(6)}$.

A acurácia de um sistema de digitalização e processamento de fotografia para o cálculo computacional do volume e profundidade de uma cavidade simulando uma UP foi avaliada em estudo em que se concluiu que foram encontrados erros mínimos e, por isso, esse sistema foi considerado um método adequado para aferição de área e volume de UP ${ }^{(7)}$.

Dentre os tipos de software disponíveis para mensuração de área de UPs, o software AutoCAD ${ }^{\circledR}$, também empregado pelos engenheiros civis no cálculo de área física em topografia, tem

sido utilizado por profissionais das áreas de oftalmologia ${ }^{(8,9)}$, ${\text { otorrinolaringologia }{ }^{(10)} \text {, odontologia }}^{(11,12)}$ e para mensuração de área de lesões cutâneas ou UPs em estudos com animais ${ }^{(13)}$ e humanos ${ }^{(1,14)}$, sendo considerado um método de medida de alta precisão e confiabilidade ${ }^{(8)}$.

O software Motic tem sido igualmente utilizado para calcular a área de UPs por meio de imagens digitais. Khalil et $\mathrm{al}^{(15)} \mathrm{e}$ Aramwit e Sangcakul ${ }^{(16)}$ calcularam a área de feridas em ratos, enquanto Silva et $\mathrm{al}^{(17)}$ descreveram elevada fidedignidade inter e intra-examinador no cálculo de área de UPs em humanos.

Portanto, tanto o software Motic quanto o AutoCAD ${ }^{\circledast}$ podem ser utilizados para mensuração de área de UPs. Entretanto, em recente levantamento bibliográfico, não foram encontrados estudos comparativos do desempenho desses recursos computacionais para identificação de propriedades geométricas de UPs. Almejando contribuir para o desenvolvimento de métodos quantitativos de avaliação em saúde, este estudo compara as aferições de área de UPs por meio desses recursos computacionais para verificar sua precisão e reprodutibilidade.

\section{MÉTODO}

O protocolo experimental do presente estudo foi aprovado pelo Comitê de Ética em Pesquisa da Fundação de Ensino e Pesquisa em Ciências da Saúde sob o Nº CEP/FEPECS 217/10. Todos os participantes receberam informações sobre o objetivo e o procedimento do estudo e assinaram um termo de consentimento livre e esclarecido no momento da coleta de dados, concordando em participar da pesquisa.

Foi realizado um estudo transversal em que foram selecionadas duas imagens de UPs com grau III de pacientes internados no Hospital de Apoio de Brasília, unidade integrante da Secretaria de Estado de Saúde do Distrito Federal (SES-DF). A primeira imagem, de contorno regular, corresponde à região trocantérica direita do paciente AMS (Figura 1) e a segunda imagem, de contorno irregular, à região isquiática da paciente BHN (Figura 2). As fotografias utilizadas no estudo foram autorizadas pelos pacientes por meio de um termo de consentimento livre e esclarecido. As imagens foram obtidas por uma

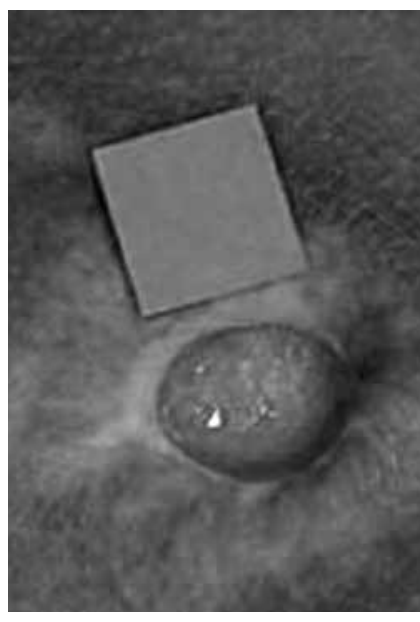

Figura 1. UP do paciente AMS Fonte: Dados dos autores

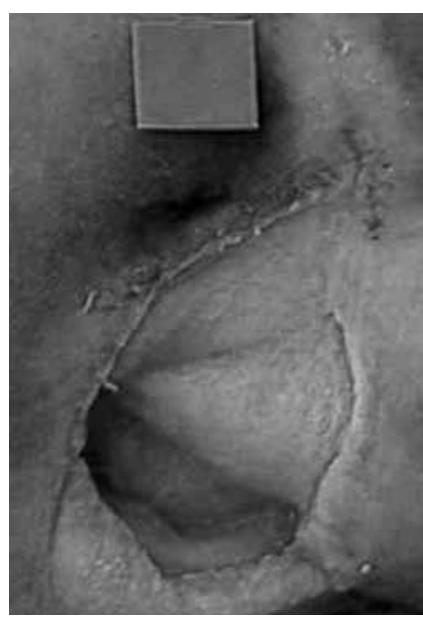

Figura 2. UP da paciente $\mathrm{BHN}$ Fonte: Dados dos autores 
câmera fotográfica digital da marca Sony Cyber-shot 5.0 megapixels, modelo DSC-V1, com resolução de 1.2 megapixels. A câmera foi posicionada com eixo perpendicular ao leito da UP e com distância de 50 centímetros, aferida com uma régua plástica. Como referência para o cálculo computadorizado da área, um objeto padrão, com área de $4 \mathrm{~cm}^{2}$, foi posicionado próximo à borda da UP.

As imagens em formato jpeg foram importadas pelo software AutoCAD ${ }^{\otimes}$ versão 2008 e pelo software Motic Images Plus versão 2.0, instalados em um notebook marca HP Pavilion modelo DV6232 com um mouse externo marca Clone modelo 6195.

Foi utilizada uma amostra por conveniência de 35 acadêmicos do curso de Fisioterapia da Universidade Católica de Brasília (UCB), sendo 13 indivíduos do sexo masculino $(37,14 \%)$ e 22 indivíduos do sexo feminino $(62,86 \%)$, com média de idade de 23,68 \pm 2,06 anos, todos possuindo conhecimento básico do Microsoft Office. Cada voluntário realizou três aferições em cada uma das imagens para a obtenção de um valor médio, inicialmente com o programa Motic e, posteriormente, com o programa AutoCAD ${ }^{\circledR}$.

\section{Determinação da área com o software Motic}

As imagens das duas UPs inseridas na área de trabalho foram ampliadas com aproximação de 200x, utilizando-se o recurso de lupa, gerando-se uma janela de melhor visualização aos avaliadores. Logo após, foi selecionada a ferramenta "irregular" e com esta, cada avaliador marcou o contorno do objeto padrão, pressionando o botão esquerdo do mouse enquanto realizava o contorno. Foi realizado o mesmo procedimento para o contorno da imagem de cada UP. Ao se finalizar o contorno do objeto padrão e da UP, o software forneceu dados referentes às suas áreas, com unidade de medida em Squm (unidades de medidas quadradas).

\section{Determinação da área com o software AutoCAD ${ }^{\circledR}$}

Aqui as imagens das UPs foram inseridas por meio dos comandos insert e raster image reference, com seleção da escala 1. Na área de trabalho do software, foi ampliado o tamanho da imagem, de modo a facilitar a demarcação do contorno. Foi selecionada a ferramenta spline para delimitar o contorno do objeto padrão e da imagem de UP e, por meio do botão esquerdo do mouse, o avaliador marcou o contorno do objeto padrão e da imagem de UP, clicando em vários pontos seqüencialmente. Ao se finalizar a demarcação, a tecla < enter $>$ foi utilizada 3 vezes consecutivas, formando o contorno definitivo do desenho. Os comandos AA (area) <enter > e $\mathrm{O}$ (object) <enter> foram utilizados para a determinação da área do objeto padrão e da imagem de UP, em números decimais.

Conversão das áreas fornecidas pelo software Motic e pelo AutoCAD ${ }^{\circledast}$

Com as áreas obtidas, foi elaborada uma tabela no software Microsoft Excel contendo a identificação do candidato e a análise do objeto padrão e da UP de cada imagem em cada software. Considerando que a área do objeto padrão era conhecida $\left(4 \mathrm{~cm}^{2}\right)$, a área UP em $\mathrm{cm}^{2}$ foi obtida por regra de três, por meio da seguinte fórmula:

$$
\text { UP } \mathrm{cm}^{2}=\frac{\text { Área da UP informada pelo software } \times 4}{\text { Área do objeto padrão informada pelo software }}
$$

\section{Análise estatística}

Para o tratamento estatístico dos dados foram utilizados média, desvio-padrão, coeficiente de variação (CV), erro das médias, teste $t$ pareado e correlação de Pearson, calculados no SPSS 15.0 (Statistical Package for the Social Sciences) para Windows, para comparar o software Motic Images Plus 2.0 e o AutoCAD 2008. A concordância entre os dois métodos foi testada utilizando o procedimento de Bland e Altman. A reprodutibilidade foi aferida por meio do Coeficiente de Correlação Intra-classe (ICC). A correlação foi considerada baixa para ICC $<0,40$, moderada para $0,40 \leq \mathrm{ICC}<0,75$ e excelente para ICC $>0,75$. Consideraram-se diferenças significativas quando $p<0,05$.

\section{RESULTADOS}

Analisando os resultados da mensuração de área das UPs (Tabela 1), pode-se observar diferença estatisticamente significativa [t(35) $=-2,30 ; p=0,03$ ] entre o software Motic e o AutoCAD ${ }^{\circledR}$ no que diz respeito à UP do paciente AMS (UP AMS), sendo que os valores de área da UP foram maiores no Motic do que no AutoCAD ${ }^{\circledR}$. Entretanto, a diferença entre as médias das áreas foi de apenas $0,08 \mathrm{~cm}^{2}(2,49 \%)$. Em relação à UP da paciente BHN (UP BHN), não se observa diferença estatisticamente significativa $[\mathrm{t}(35)=1,07 ; \mathrm{p}=0,29]$ entre os dois tipos de software, com diferença entre as médias das áreas de apenas $0,22 \mathrm{~cm}^{2}(1,05 \%)$. O valor máximo do coeficiente de variação encontrado foi de $6,08 \%$.

Os valores da correlação de Pearson demonstraram correlação positiva e fraca $(r=0,34 ; p=0,04)$ entre os softwares Motic e AutoCAD ${ }^{\circledast}$ na mensuração da UP AMS e nenhuma correlação estatisticamente significativa entre as duas medidas $(r=0,06 ; p=0,75)$ da UP BHN. Em relação ao ICC referente às duas medidas, observou-se valor muito baixo $(\mathrm{ICC}=$ $0,16)$ na UP AMS e valor moderado $(I C C=0,41)$ na UP BHN.

O gráfico de Bland e Altman apresenta a concordância entre a mensuração das UPs com o software Motic e o AutoCAD ${ }^{\circledR}$. No eixo y estão as diferenças individuais em função das médias observadas nos dois métodos (eixo x). No Gráfico 1, a linha central representa a média da diferença da mensuração da UP AMS entre os dois métodos $(0,08 \pm 0,21$ $\mathrm{cm}^{2}$ ). Para definir os limites superior e inferior utilizou-se uma fórmula (média $\pm 1 x$ desvio-padrão), resultando em limite superior de $0,29 \mathrm{~cm}^{2}$ e limite inferior de $-0,13 \mathrm{~cm}^{2}$. Dessa forma, permitiu-se um erro de aproximadamente $0,2 \mathrm{~cm}^{2}$ de diferença entre um teste e outro. No Gráfico 2, a linha central representa igualmente a média da diferença da mensuração da UP BHN entre os dois métodos $\left(0,22 \pm 1,23 \mathrm{~cm}^{2}\right)$. O limite superior e o limite inferior foram de $1,45 \mathrm{~cm}^{2} \mathrm{e}-1,01 \mathrm{~cm}^{2}$, respectivamente. Dessa forma, permitiu-se um erro de aproximadamente $1 \mathrm{~cm}^{2}$ de diferença entre um teste e outro. 
Tabela 1 - Análise estatística descritiva da mensuração das UPs dos pacientes AMS e BHN utilizando os softwares Motic e o AutoCAD

\begin{tabular}{|c|c|c|c|c|}
\hline \multirow[t]{2}{*}{ Grandezas } & \multicolumn{2}{|c|}{ UP AMS } & \multicolumn{2}{|c|}{ UP BHN } \\
\hline & Motic Plus 2.0 & AutoCAD 2008 & Motic Plus 2.0 & AutoCAD 2008 \\
\hline Média \pm desvio padrão $\left(\mathrm{cm}^{2}\right)$ & $3,29 \pm 0,20$ & $3,21 \pm 0,16$ & $20,99 \pm 1,11$ & $21,21 \pm 0,58$ \\
\hline CV (\%) & 6,08 & 4,98 & 5,29 & 2,73 \\
\hline Valor $\mathrm{t}$ & \multicolumn{2}{|c|}{$-2,30$} & \multicolumn{2}{|c|}{1,07} \\
\hline Valor $p$ & \multicolumn{2}{|c|}{$0,03 *$} & \multicolumn{2}{|c|}{0,29} \\
\hline Erro das médias (\%) & \multicolumn{2}{|c|}{2,49} & \multicolumn{2}{|c|}{1,05} \\
\hline
\end{tabular}

*Diferença estatisticamente significativa.

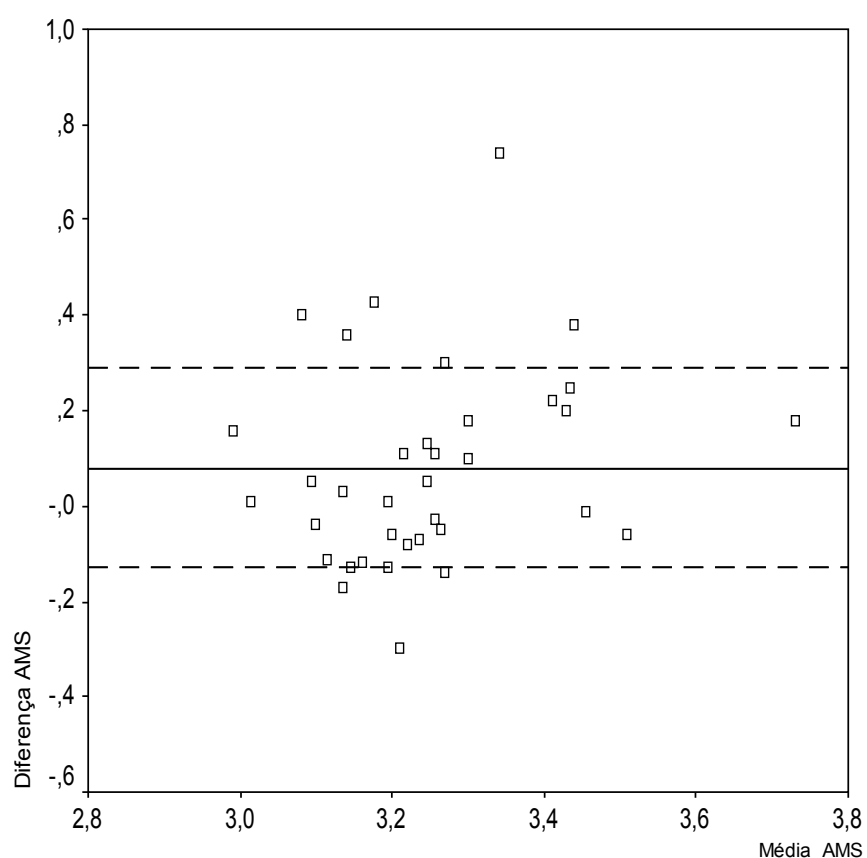

Gráfico 1. Gráfico de Bland e Altman da UP do paciente AMS.

\section{DISCUSSÃO}

Foi observada baixa consistência dos resultados, haja vista que houve diferença estatisticamente significativa apenas em uma das UPs, baixa correlação e reprodutibilidade, porém houve concordância entre os métodos. Estes resultados podem ter sofrido influência de fatores como a coordenação motora dos avaliadores, noção diferenciada da delimitação da borda da UP, diferentes tamanhos das UPs, tipo de borda a ser delimitada e características técnicas dos dois tipos de software. Para minimizar essa influência, a mensuração da área da UP deveria ser realizada, preferencialmente, pelo mesmo sujeito e software.

Observa-se que a área da UP da paciente BHN é aproximadamente sete vezes maior do que a do paciente AMS e a técnica de

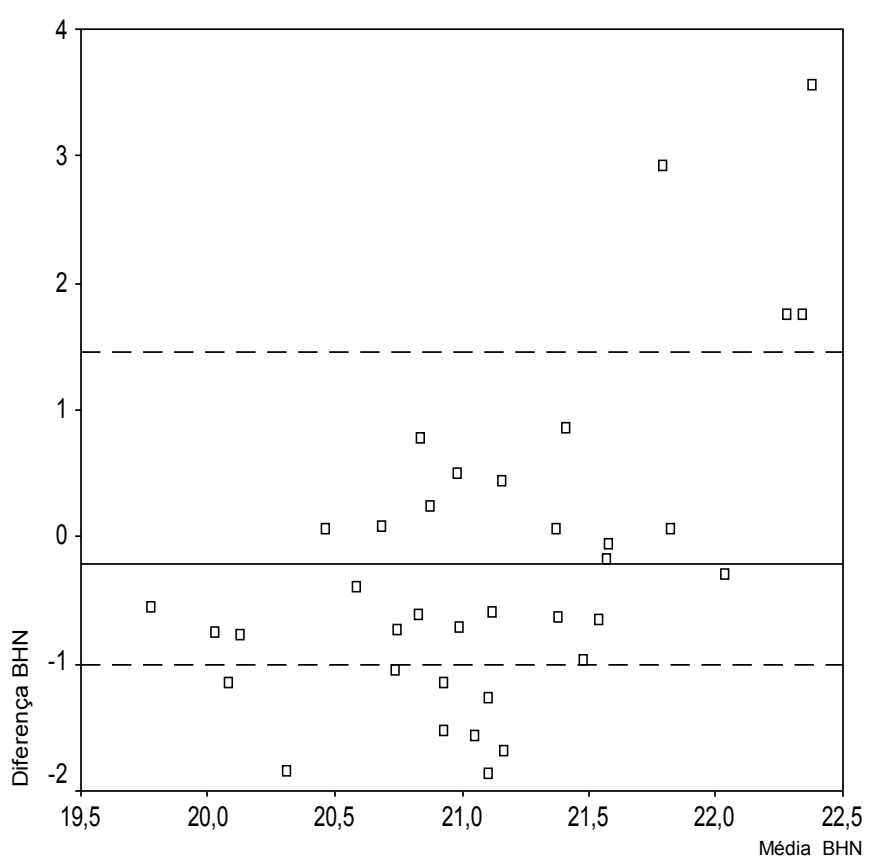

Gráfico 2. Gráfico de Bland e Altman da UP da paciente BHN.

marcação utilizada requer deslocamentos da mão do operador, cujos desvios, do ponto de vista absoluto, independem do tamanho da figura percorrida. Entretanto, do ponto de vista relativo, tais desvios ficam mais acentuados em figuras de menor área.

Embora tenha sido encontrada baixa consistência, o CV máximo foi de 6,08\%, estando abaixo do valor máximo de $11,34 \%$ descrito por Silva et $\mathrm{al}^{(13)}$ ao avaliar UPs com o software Motic. Segundo Gomes ${ }^{(18)}$, os valores de CV encontrados no presente estudo são considerados baixos, caracterizando ambos os métodos como de alta precisão.

Apesar de ter ocorrido erro de $2,49 \%$ para a UP do paciente AMS e de 1,05\% para a UP da paciente BHN entre as médias do software Motic e do AutoCAD ${ }^{\circledast}$, estes valores são considerados pequenos principalmente do ponto de vista 
clínico, pois constituem erros bem menores que os cometidos pelos métodos simples de mensuração de área como a régua (comprimento x largura).

Os resultados obtidos nesta pesquisa não puderam ser comparados com os de outros estudos por não terem sido encontrados similares na literatura. Sugere-se que novos estudos sejam realizados comparando os dois tipos de software, analisando UPs com diferentes profundidades, tamanhos e tipos de borda.

\section{CONSIDERAÇÕES FINAIS}

O estudo alcançou o objetivo proposto, tendo permitido concluir que ambos os instrumentos apresentam pequenos erros de medida. Apesar de haver diferença estatística e baixa reprodutibilidade, eles concordam entre si, sendo mais confiáveis e precisos do que técnicas simples de mensuração de área, permitindo assim um melhor acompanhamento da evolução da cicatrização das UPs.

\section{REFERÊNCIAS}

1. Mendes AFO. Avaliação do laser, com o comprimento de onda $670 \mathrm{~nm}$, no processo de cicatrização de úlceras de pressão no paciente lesado medular. Brasília. Dissertação [Mestrado em Ciências da Saúde]- Universidade de Brasília; 2002.

2. Camargo SMPLO. Estudo combinado no ultra-som pulsado de baixa intensidade e da papaína na cicatrização de úlcera por pressão no atendimento domiciliar. São Paulo. Dissertação [Mestrado em Bioengenharia]- Universidade de São Paulo; 2006.

3. Sousa CA, Santos I, Silva LD. Aplicando recomendações da Escala de Braden e prevenindo úlceras por pressão evidências do cuidar em enfermagem. Rev Bras Enferm 2006;59(3):279-84.

4. Castilho LD, Caliri MHL. Úlcera de decúbito e estado nutricional: revisão da literatura. Rev Bras Enferm 2005;58(5):597-601.

5. Ministério da Saúde (Brasil) Secretaria de Políticas de Saúde. Departamento de Atenção Básica. Manual de condutas para úlceras neurotróficas e traumáticas. Ministério da Saúde: Brasília; 2002.

6. Krouskop TA, Barker R, Wilson MS. A noncontact wound measurement system. J Rehab Res Develop 2002;(39):337-46

7. Lopes AG, Soares MC, Santana LA, Guadagnin RV, Neves RS. Aferição não-invasiva de úlcera por pressão simulada em modelo plano. Rev Bras Enferm 2009;62(2):200-3.

8. Sánchez Pérez A, Honrubia López FM, Larrosa Poves JM, Polo Llorens V, Melcon Sánchez-Frieras B. Sistema AutoCAD para el estudio planimétrico de la papila óptica em el glaucoma: técnica y estudio de reproducibilidad. Arch Soc Esp Oftalmol 2001;76(9):551-8.

9. Almeida CAM, Schellini SA, Gregório EA, Pellizzon $\mathrm{CH}$. Utilização do AutoCAD 2004 para quantificação de pesquisas usando fotomicrografias eletrônicas. Rev Bras
Oftalmol 2007;66(4):227-30

10. Terheyden $\mathrm{H}$, Maune S, Mertens J, Hilberg O. Acoustic rhinometry: validation by three-dimensionally reconstructed computer tomographic scans. J Appl Physiol 2000;(89):1013-21.

11. Goiato MC, Vedovatto E, Amantéa DCZ, Gennari Filho H, Marinho MLVD. Análise da movimentação dos dentes artificiais em próteses totais superiores. Influência do tipo de polimento. Ciênc Odontol Bras 2006;9(1):6-16.

12. Constante IGT, Davidowicz H, Barletta FB, Moura AAM. Study of the areas and thicknesses of mesiobucal root canals prepared by three endodontic techniques. Braz Oral Res 2007;21(2):118-26.

13. Garros IC, Campos ACL, Tâmbara EM, Tenório SB, Torres OJM, Agulham MA, et al. Extrato de Passiflora edulis na cicatrização de feridas cutâneas abertas em ratos: estudo morfológico e histológico. Acta Cir Bras 2006;21(3):55-65.

14. Echeverría JM. Validación de un nuevo método de análisis digital de superficies. Cir Plas Iberolatinoam 2006;32(2):71-82.

15. Khalil EA, Afifi FU, Al-Hussaini M. Evaluation of the wound healing effect of some Jordanian traditional medicinal plants formulated in Pluronic F127 using mice (Mus musculus). J Ethnopharmacol 2007;109(1):104-12.

16. Aramwit $P$, Sangcakul A. The effects of sericin cream on wound healing in rats. Biosci Biotechnol Biochem 2007;71(10):2473-7.

17. Silva SF, Rocha JR EF, Neves RS, Santana LA, Guadagnin RV. Fidedignidade inter e intra-examinador do cálculo de área de úlceras por pressão em fotografias utilizando o software Motic. Brasília. Monografia [Graduação em Fisioterapia]- Universidade Católica de Brasília; 2007.

18. Gomes FP. Curso de estatística experimental. 14. ed. Piracicaba: Degaspari; 2000. 